\title{
EBIC-Enabled NanoManipulators - Investigating Dislocations in mc-Solar Cells
}

\author{
P. Hamer ${ }^{1}$, D. Tweddle ${ }^{1}$, T. Martin ${ }^{1}$, P.Wilshaw ${ }^{1}$, M. Hiscock ${ }^{2}$, and J. Lindsay ${ }^{2}$ \\ 1. Oxford Instruments NanoAnalysis, Halifax Road, High Wycombe, UK \\ 2. Department of Materials, University of Oxford, Oxford, UK
}

Multicrystalline silicon (mc-Si) solar cells are an important and cost effective technology with a significant role to play in the mix of energy sources required in the future. Recent advances have led to industrial champion cell efficiencies of over $21 \%$ [1], however typical industrial cell performance is much lower, in large part due to variability in the distribution of recombination centers between mc-Si wafers. These recombination centers include impurity decorated grain boundaries and dislocations [2, 3], and act to reduce the efficiency of the solar cell by reducing the number of light generated minority carriers which are collected at the p-n junction. As such, an understanding of these defects - the elements which decorate them, their recombination activity and what processes can reduce this activity, is essential if improvements are to be made.

Photoluminescence imaging [4] can be used to investigate the minority carrier lifetime of solar cells on a macro scale but the method is not suitable when there is a need to investigate individual dislocations and their effect on the efficiency of the cell. These dislocations exist on a scale of nanometres meaning that there is a significant gap in scale between the minority lifetime mapping provided by photoluminescence and the features which have a significant effect on that lifetime. Electron Beam Induced Current (EBIC) is an SEM/FIB based technique which utilizes an incident electron beam to induce currents in semiconductor samples $[5,6]$. As the beam is rastered over the sample the generated current will vary with the structures that are scanned. This variation can be detected and measured by connecting the sample to an amplification system which is able to associate the current with the beam position to produce images where the contrast indicates the degree of current flow - in this application this enables imaging of recombination activity of dislocations.

Here we present a method which utilizes a nanomanipulator which has been combined with an EBIC system to both identify dislocations and lift out samples for subsequent analysis. By utilizing the same nanomanipulator for both the EBIC characterization of the sample and liftout of dislocations, handling is reduced and workflow speed and ease is greatly improved. Capital expenditure on equipment is also reduced as only one piece of equipment is needed for both tasks. The identification and liftout of a dislocation can be achieved in a single analysis session.

P-type mc-Si samples were prepared through chemo-mechanical polishing of the surface to be imaged. This was followed by a Secco etch [ref] to reveal dislocations through the formation of etch pits. The samples were then RCA cleaned and $13 \mathrm{~nm}$ of aluminium was thermally evaporated on the front surface to form a Schottky contact. The samples were then mounted on a sample stub using indium-gallium and silver dag to provide good ohmic contact between the rear surface of the sample and the FIB stage. The stage and nanomanipulator (Oxford Instruments OmniProbe 200) (fitted with a coaxial shaft) were connected to the EBIC amplifier (Oxford Instruments/Point Electronic DISS5 system). Whilst imaging with the e-column, the nanomanipulator was placed on the Schottky contact. The scanning of the ebeam introduces charge to the sample and induces a current in the solar cell - an Electron Beam Induced Current. Figure 1 shows a representation of this setup as it was used with this sample. The current 
within this circuit is measured at each point of the beam's raster to form a map of current flow within the sample which is the EBIC image (Figure 2). While the etch pits are clearly visible on the image it is also possible to observe the recombination associated with these dislocations.

It was noted from Figure 2 that some dislocations had a significantly higher EBIC contrast than others. It is this difference that is of particular interest for further study. Several dislocations were chosen for further analysis and the associated etch pits were filled with tungsten using a gas injection system. A carbon protection layer was also deposited. A trench was then milled around the sample to create a lamella. This was then lifted out with the nanomanipulator and welded to a TEM half grid (Figure 3). Once mounted it was then available for further analysis by other methods.

\section{References:}

[1] Green, M.A., et al, Progress in Photovoltaics 25 (2016) (NREL/JA--5J00-67687).

[2] Schindler, F., et al, Energy Procedia 77 (2015), p. 633.

[3] Stokkan, G., et al, Solar Energy Materials and Solar Cells 130 (2014), p. 679.

[4] Trupke, T., et al, Applied Physics Letters 89(4) (2006), p. 044107.

[5] Fell, T., P. Wilshaw, and M. De Coteau, physica status solidi (a) 138(2) (1993), p. 695.

[6] Wilshaw, P. and T. Fell. Proceedings of the 6th International Symposium on the Structure and Properties of Dislocations in Semiconductors. (1989).

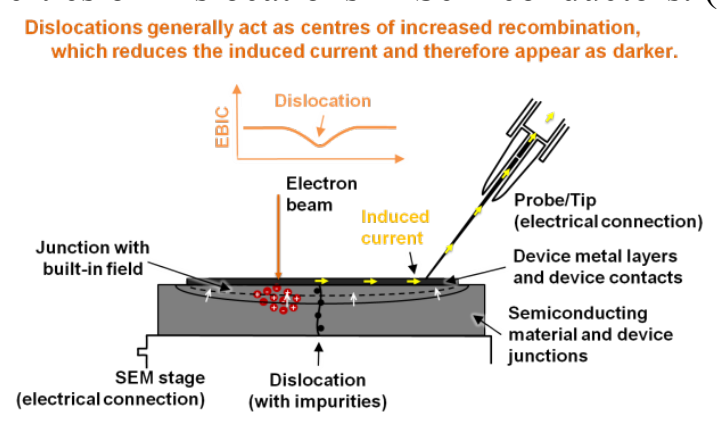

Figure 1. EBIC signal generation in Solar Cells

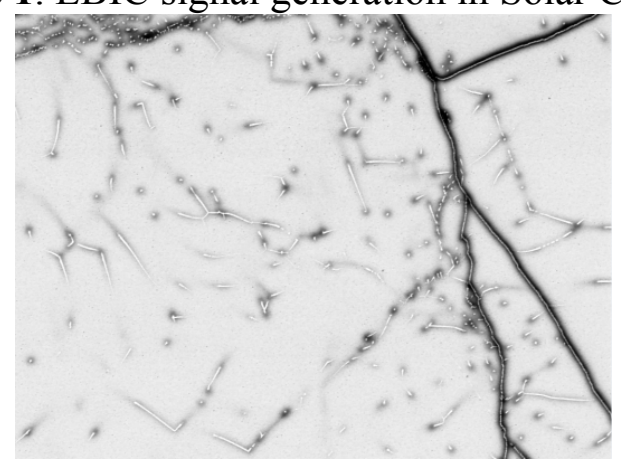

Figure 2. EBIC Image of the mc-Si Solar Cell

Dislocation etch pits appear as white, recombination activity as grey/black.

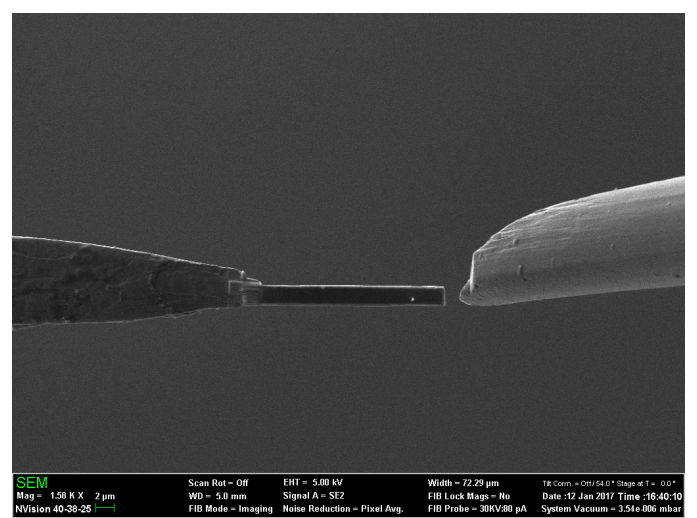

Figure 3. The lifted out lamella after attachment to a TEM half-grid

The dislocation appears as a white spot on the visible face of the lamella. 